\title{
Speculative Method and Twitter: \\ Bots, Energy and Three Conceptual Characters
}

Alex Wilkie

Goldsmiths, University of London

Mike Michael

The University of Sydney

Matthew Plummer-Fernandez

Goldsmiths, University of London

\begin{abstract}
This paper aims to contribute to recent innovations in social scientific methodology that aspire to address the complex, iterative, and performative dimensions of method. In particular, we focus on the becoming-with character of social events, and propose a speculative method for engaging with the notas-yet. This work, being part of a larger project that uses speculative design and ethnographic methods to explore energy-demand reduction, specifically considers the ways in which energy-demand reduction features in the Twitter-sphere. Developing and deploying three automated Bots whose function and communications are at best obscure, and not uncommonly nonsensical, we trace some of ways in which they intervene and provoke. Heuristically, we draw on the 'conceptual characters' of idiot, parasite and diplomat in order to grasp how the Bots act within Twitter to evoke the instability and emergent eventuations of energy-demand reduction, community, and related practices. We conclude by drawing out some of the wider implications of this particular enactment of speculative method.
\end{abstract}

Keywords: Twitter, energy-demand reduction, speculative methods, digital methods, software roBots, Bots, performativity, science and technology studies, parasite, idiot, diplomat. 


\section{Speculative Method and Twitter: Bots, Energy and Three Conceptual Characters}

\section{Introduction}

In recent sociological thought, there has been an increasing concern with what precisely comprises the empirical and the methods used to access this. It has now become commonplace to note the performative dimension of sociology's methods: they are constitutive of the object they 'study'. For instance, along with numerous scholars, we might ask: What or who is being 'othered' during empirical research? Further, taking a process-oriented perspective, we might also ask: If empirical research can be thought of as an event in which the component elements (including the researcher) co-become, how is the research phenomenon to be understood when it is constitutively open? In other words: how do we access this immanent or virtual dimension of the social event - not least that social event known as the sociological empirical study? How, indeed, do we shift from seeing research as answering a research question to seeing it as the opportunity for asking more inventive questions?

This paper attempts to contribute to this broad empirical and analytic strategy in a number of ways. Firstly, we are interested in social phenomena that arise on Twitter. We will elaborate on the importance of this domain below. Suffice to say, here, that we are concerned with the complex patterns of communication on Twitter and the prospective emergence of new, prominent concerns or issues from particular rounds of exchange.

Secondly, in wanting to engage communications mediated by Twitter, we make direct use of the performativity of method. Specifically, we develop techniques that attempt to engage with the prospects of, and what is immanent in, the exchanges that take place on Twitter. We go about this by prompting Twitter responses through the use of software research robots (henceforth Bots), which contribute in routinely odd and contrary ways to the normal flow of exchanges. Bots are therefore methodological interventions that are overtly constitutive of the material that is gathered, but in ways that are open, ambiguous or troublesome. In triggering such responses, the aim is to access new and emergent formulations of the 'issues at stake' in Twitter exchanges.

Thirdly, the introduction of Bots as a performative methodological technique - or, more accurately, as speculative devices - takes place within the substantive area of energy-demand reduction. The current work is part of a larger interdisciplinary project that combines science and technology studies and so-called 'speculative design' to produce a computational and interactive device that will be given to UK-based 'energy communities.' The overarching aim is to enable a re-thinking and a re-enactment of the issues around, and engagement with, energy-demand reduction. Our engagement with Twitter through software Bots is thus aimed at both exploring online energy-demand reduction actors as well as generating communications that will be mediated by the 'Energy-Babble' device. So, insofar as the Bots are part of a wider project of accessing emergent enactments of the 'issues at stake', those issues are generally concerned with 'energy-demand reduction', though, of course the Bots are designed to provoke a rethinking and re-enactment of the very parameters of energy-demand reduction itself, including what can count as relevant actors, communities and practices. 
In what follows, we firstly outline in more detail the conceptual and theoretical underpinnings of the paper. We then go on to describe the project itself, and the role of the Bots. The sort of material generated through the operation of the Bots is subsequently considered. In light of this, we rethink the status of the Bots through a number of heuristic 'conceptual characters': namely the idiot, the diplomat and the parasite. We conclude with some reflections on the utility of Bots, as well as on some of their disadvantages.

\section{The Performativity of Method and the Web}

A burgeoning trend within social and cultural research concerns the doing of method and its rejuvenation (see for example the recent volumes: Adkins and Lury, 2012; Back and Puwar, 2012; Lury and Wakeford, 2012) in the face of the changing patternings of sociocultural life. Notably, such interest emphasises the role of information technologies that contribute to the pervasive production and harnessing of social data, and the challenges this presents for analysis as well as the accountability and governance of data and datasets. Within such debates, the web presents both methodological opportunity as well as rendering problematic the doing of sociology 'as usual'. Thus, on the one hand scholars are involved in efforts to develop 'digital devices' and techniques that engage the web, whether through the visualisation of co-link analysis (e.g. Weltevrede and Helmond, 2012), the analysis of tag clouds (e.g. Rogers, 2010: 253), the study of search queries (Rogers, 2013: 104) and the scraping of search engine returns (Rogers, 2009), the analysis of Twitter metatagging (Marres and Weltevrede, 2012; e.g. Shah and File, 2011) or samples (Gerlitz and Rieder, 2013). On the other hand, and in the context of 'knowing capitalism' (Thrift, 2005), there is routine widespread, large-scale data harvesting and analysis conducted by innumerable commercial organisations. In this light, Savage and Burrows (2007) argue that sociology must revitalise its interest in methodological innovation, if only to resist conceding relevance, competence and expertise to actors with principally commercial interests. For scholars interested in 'big data' (Boyd and Crawford, 2012), such empirical material is seen to provide ways of relating individuals to population (Ruppert, 2011) by processing indicators of actual practices (Ruppert and Savage, 2011: 84; Webber, 2009: 176). ${ }^{1}$ We are sympathetic to such approaches, and, accordingly, we would wish to acknowledge Web 2.0 as a setting for particular practices, as well as a domain that is co-configured by such practices (in contrast to regarding Web 2.0 as merely indicative of offline activities). We are also keen to argue that the sorts of entities that inhabit Twitter are ontologically varied and complex and thus irreducible to individuals, human collectives, or human communication. In the present paper we contribute to such methodological and conceptual innovation (see also: Crompton, 2008), specifically taking a performative approach which, by way of speculative design, seeks to develop novel methods that engage with dynamics and potentiality of Twitter in relation to energy demand reduction.

John Law (2004) provides a powerful argument that social scientific methodology is inherently performative. A method (or 'method assemblage') will involve "the crafting and enacting of boundaries between presence, manifest absence and Otherness' (2004: 85). As such particular types of 'data' are excluded - they fail to register for the researcher - by virtue of falling outside a method's frame. However, 'non-sensical' other-ed data can nevertheless impinge on the event of research. Stengers 
(2005) has raised the profile of a potential producer of such 'nonsensical data', namely the philosophical character of the 'idiot'. We shall have much more to say about this figure below. Suffice it to say that this and similar figures (e.g. parasite, diplomat, trickster, coyote) can serve as markers for ways in which we might sensitize ourselves to the actions, talk, objects and organisations that make no sense for the research event at hand. Such sensitization - listening to these unintelligible murmurings - can enable us to become suspicious that we, as researchers, are in possession of "the meaning of what we know", as Stengers (2005: 995) puts it.

Drawing on Whitehead (1978) we can say that events entail a range of elements that come together and combine to constitute, in this case, the research event. This event can be understood in two contrasting ways according to Fraser (2010). Either the elements are stable and co-exist with - be-with - one another; or they mutually affect - co-become with - one another. If we adopt the latter option, then we also need to address the fact that the event is far less easy to grasp empirically: as the elements change, so too does the nature of the event. It becomes other to itself, so to speak - it becomes underdetermined, opening out to a range of possible potentials or virtualities. The function of the analyst itself becomes something other: one of accessing and exploring these possibilities. Put another way, methodology becomes less a case of answering a pre-known research question (why do people fail to engage energy-demand reduction) than a process of asking inventive, that is, more provocative questions where intervention stimulates latent social realities, and thus facilitate the emergence of different questions (c.f. Lezaun et al., 2013: 3). Such enquiries might address the meaning of 'energy', the staging of 'practice' or the parameters of studying energy 'consumers' and 'communities'.

So if our methods affect the emergence of a social event, and if this event is conceived as open and unfolding, we can pursue a pro-active process of 'speculatively intervening' in such events. Specifically, we can interject ambiguous, opaque and even nonsensical elements that allow for the possibilities or virtualities of those events to become 'apparent'. Michael (2012a, 2012b) has suggested that the methods of Speculative Design are one means of doing this. Speculative Design, with its ambiguous, playful probes and devices, attempts to test the limits of what interaction design can do. In social scientific terms, the fact that the function of these objects doesn't make sense - is obscure or ludic means that their users begin to relate to certain social events or phenomena in unusual or unexpected ways. For instance, users of the 'Local Barometer' can move toward a version of the neighbourhood that is not simply an economic, social and cultural category. The Local Barometer electronically feeds web-based advertisements into the home. The distance and direction of these advertisements is affected by wind speed and direction (measured by a locally-installed anemometer). When the participant starts reading the weather through the advertisements, then the neighbourhood becomes something altogether more heterogeneous, incorporating the meteorological and the electromagnetic as well as the cultural and the economic (Michael and Gaver, 2009). Here, we would argue, the Local Barometer facilitates unexpected practices that reformulate the question of the neighbourhood: it becomes something that is technonatural, or naturecultural as opposed to simply cultural or economic.

In this paper, we align ourselves with this broad speculative project, producing speculative devices to explore the dynamics of Twitter. In particular, we introduce Bots that address the Twitter-sphere of energy-demand reduction. These Bots share the characteristic of speculative devices in that their functionality is not always clear: the 'functions' they perform - the communications that they produce - 
do not necessarily make much sense in the context of the energy-demand reduction Twitter-sphere. The purpose of this paper is thus to explore the extent to which these Bots have enabled us speculatively to access and engage this Twitter-sphere's possibilities and virtualities.

First, however, we outline the project of which the Bots are a part, and give some background to their development and programming.

\section{Engaging Twitter as a medium for energy-demand reduction}

Sustainability Invention and Energy-demand reduction: Co-Designing Communities and Practice (ECDC for short) is one of seven projects funded under the Research Councils United Kingdom's (RCUK) Energy Programme. ${ }^{2}$ The aim of this programme is to investigate "How individuals and communities use energy, their understanding of energy use and effective, community management of energy and energy regulation" in order to inform and support the meeting of the UK energy and environmental targets, in particular carbon reduction initiatives as set and out and undertaken by the UK government. ${ }^{3}$ In brief, ECDG addresses energy-demand reduction within the context of communities of practice by raising issues about the nature of social and technical practices. While in common with others, we see practices as deeply embroiled with community (Shove et al., 2012: 68; e.g. Wenger, 1998), we also feel that both terms carry certain assumptions. As such, the project addresses a range of issues, including: the relation of practices to senses of 'ownership' of, or commitment to, relevant technologies and communities; the enactment of collective practices around relevant technology; and the aesthetic, playful, affective, creative, ambiguous aspects of practice which play a role in energy-demand reduction.

The general term we have developed to capture our approach is 'ludic action research' (see Gaver, 2002) in which broadly ethnographic techniques iteratively combine with speculative design. Accordingly the aim is to resource participants' practices in relation to the issues of energy-demand reduction in ways that are affective, playful, exploratory and prospective as well as practical and political. The ultimate aim is to stimulate the participants to re-frame the 'problem' of energy-demand reduction.

Put another way, our aim is to develop a design that sets up the possibility of a 'cosmopolitical event' (e.g. Stengers, 2005). This speculative device - the 'Energy-Babble' - needs to be sufficiently open, playful, intriguing, ambiguous, complex, and beautiful that it will engage its users (the members of local energy communities) so that they come to question what is meant by 'energy' (let alone energydemand reduction), and to query what 'community' might mean (let alone an energy community). More specifically, the Energy-Babble is a radio-like sound device that vocalises and amplifies energyrelated content drawn from the web (including Twitter and UK electricity grid updates) and combines this with voice and SMS messages inputted by the community members. The Twitter-derived content is partly generated by the Bots discussed in this paper. Thus, the Energy-Babble seeks to combine local expertise in, and views about, energy-demand reduction with live updates from news media, government agencies, other communities, commentators, activists, and environmental scientists. The result is a stream of energy babble that often does not make immediate sense.

In this context, our rationale for taking to Twitter was informed by the observation that the web has 
become an increasingly important and 'live' setting in which community-based initiatives and energy demand reduction efforts are enacted (see Java et al., 2007). On this score, Twitter is part of a cascade of mechanisms for circulating environment related news media, publicity and information as well as a domain in which energy community identities and markers of practice are rendered public and made accountable. In addition, as researchers interested in Web 2.0 and digital methods argue, the web is a setting for the mediation of matters of concern (e.g. Marres and Rogers, 2005). Here, Twitter exemplifies the capacity and increasing prevalence of online social networking platforms to mediate such happenings as disasters (Murthy and Longwell, 2012), emergencies (Hughes and Palen, 2009), public health epidemics (Goolsby, 2009), political processes (Tumasjan et al., 2010) and upheavals (Burns and Eltham, 2009). They also serve as a mechanism for the precipitation of controversies. ${ }^{4}$ So, over and above its status as an object of study through which public engagement with environmental and energy-demand issues can be investigated, Twitter is a medium through which to develop and deploy novel 'technologies of elicitation' (Lezaun and Soneryd, 2007) that occasion public responses and enact communities and publics.

\section{Twitter and Bots}

For social media scholars, Twitter is perhaps the most prominent and globally widespread form of microblogging. Launched in 2006, Twitter is a communication service that allows users to post short 'tweets' - status updates up to a maximum of 140 characters - that are chronologically organised on a visual timeline. Tweets can be posted and viewed through a number of mediums including web pages, SMS and through a variety of applications for mobile devices, including smart phones and tablet computers. One key feature of Twitter is the ability for users to 'follow' others - akin to adding them to a list of contacts - and in so doing receive message updates from those accounts which are then interspersed in their timeline. Here, users are identified by a username, proceeded by the @ symbol. For example, the UK Department of Energy and Climate Change (DECC) uses the handle @DECGgovuk. By incorporating such syntactical markup into Tweets, users can also address, respond to and cite other users as well as address a particular topic, or 'trend', which is also accessible to others, not on a user's list of followers. Users can address (Honeycutt and Herring, 2009) one another by using the @ symbol as a way to refer or cite another user (Boyd et al., 2010). Accordingly, such techniques underpin conversations on Twitter as well as enabling the means for oblique citation as this tweet from Mark Knowles, Head of Low Carbon Liverpool City Region illustrates: "Looking forward to tomorrow's British Energy Challenge. @DEGCgovuk @amandajlyne @livuni will be insightful”. Here, Mark addresses DECC, his colleague, Amanda Lyne, Chair of the Liverpool Low Carbon Economy Committee, and Liverpool University.

Although users can simply re-post others' messages - a practice known as retweeting - without indicating the source, authorship is commonly attributed by way of the letters 'RT' (ReTweet) followed by the original author's username, thus a customary denotation for a retweet would include 'RT @ username ABC' (where ABC is the content of the tweet). Here, DEGG retweets a post by the Centre for Sustainable Energy (CSE) charity: "RT @cse_communities: Check out the brand new community \#GreenDeal \& energy efficiency resources on planlocal.org.uk/planlocal-ener...". In their analysis of 
retweeting practices, Boyd et al. (2010: 10) describe variations in conversational practices on Twitter, arguing that Twitter users "inhabit a multiplicity of conversational contexts at once." Thus, in the tweet above, DECG is retweeting the CSE who are publicizing a new website for promoting sustainable community living and, by way of the syntax \#GreanDeal, addressing the message to a 'trend' or topic of interest on Twitter concerned with the UK Government's Green Deal policy for stimulating domestic energy-saving. In another example of a trend, DECG urges its followers to participate in a global energy-demand reduction event coordinated by the online community earthhour.org: "Don't forget to turn your lights off for \#EarthHour tomorrow at 8.30pm http://youtu.be/nzhKDjTFTd8 @earthhour”. Notably, the hyperlink embedded in this message connects to a YouTube video featuring the UK Minister of State for Energy and Climate Change, urging individuals to involve themselves in earthhour by modifying their 'behavior', such as switching off household electrical appliances, recycling and using public transport and thus achieve a "more sustainable, low carbon world". 5

Such Twitter conversations illustrate how tweets link up multiple conversational contexts that connect individuals with governmental and academic institutions, emerging energy communities and carbon-reduction practices in what might be characterized, to borrow from Stengers (e.g. 2005b), as an emergent environmental 'ecology of practices' mediated by Twitter - practices that are entangled with other practices, such as a government agency producing and distributing a video featuring a Minister of State on YouTube. Furthermore, and as exemplars of online energy-demand reduction practices, such Tweets also problematize how 'users' and 'communities' are characterized by existing studies of microblogging. Such studies exhibit a tendency to focus on human collectives in a specified geographic location and thereby screening out, or 'othering', hybrid (human/non-human) actors and collectives whether they be stabilized institutions, such as DECC, emergent actors, such as the community of followers contributing to \# earthhour or, for that matter, software-based actors operating on Twitter. In short, other scholarly accounts of Twitter, whether addressing communication or extensive data analytics, sift out the noise of the medium, which arguably, is one of its defining features.

In short, the Bots analyzed were coded and operated by way of combinations of off-the-shelf technologies and bespoke software. The first Bot, @Jimmy_gibbon utilized a combination of online translation services and automated posting tools in order to source and translate existing posts from English into another language, then back to English. The resulting messages were at best obscure and often nonsensical. The second Bot, @EcoJo employed a set of software algorithms to simulate the activity of a human user, not uncommon on Twitter. This provided @EcoJo with the facility to tweet, retweet and converse with other users as well as automatically add followers. @EcoJo also featured a Markov 'voice' using an algorithm for generating 'natural' text by analyzing and re-writing textual strings following grammar-like rules on sets of energy-related Twitter content. Lastly, @ErtBot, employed a very specific scraping routine where, on a regular and pre-defined basis, the Bot would conduct a search for tweets including the phrase "switched off the". Upon encountering such a tweet @ ErtBot replies to the user with a message stating "(username) has switched off the (device) and this helped to reduce our overall energy demand." Each reply then closes by way of congratulating the user or expressing enthusiasm for their environmental action using one of a number of pre-defined textual strings. Thus, @ ErtBot translates reports of device-centered practices into energy-demand reduction practices. ${ }^{6}$ 


\section{Idiots, diplomats and parasites: three conceptual characters}

So, how might we appreciate the Bots' exchanges as energy-demand reduction events that enable cobecomings and yield more inventive questions about the 'issues at stake'? To address this, we need to grasp the complexities of the Bots' workings, and to this end we heuristically employ a number of analytic figures, though mainly the idiot, the diplomat, and the parasite. These figures act as handy vehicles for analytic and speculative thought: they sensitise us to different aspects of the Bots, to the sorts of communications they set in train, and the emergent reconfigurations of the issues around energy-demand reduction. Of course, an evident irony of our approach is that in making the Bots accountable for analysis and theorisation we are in fact disciplining and ordering mess that marks the empirical world (Law, 2004; Michael, 2004). The Bots, however, were designed and deployed as provocations (within the broader ECDC design project), and the following analysis emerged in light of their interactions. We regard the conceptual characters we employ as affording a heuristic handle on the Bots' doings in Twitter and we assume there is plenty of 'mess' we do not access: the 'other' that the Bots do point to can only ever be partial.

For Deleuze and Guattari (2011), and Stengers (2005), the idiot is thus a figure who slows down thought by making no sense in its actions. It thus resists unanimity and problematizes the taken-forgranted (this is especially relevant to predominant economic models that inform energy-demand reduction). For Stengers, the idiot "resists the consensual way in which the situation is presented in which emergencies mobilize thought or action" (2005: 994). Accordingly, the idiot is connected with what Stengers calls 'cosmopolitical' events - the process of doing politics in which (non)human actors become-with one another whilst acknowledging the potential for exclusion, and insisting that 'othering' be addressed. To proceed with cosmopolitical eventuation is to exercise care, caution and sensitivity to the nature and identity of those involved in becoming through the event. Ironically, the utility of the idiot in analytic terms lies in its potential capacity to remain indifferent to other's concerns, not least those of the researcher. For Stengers, the idiot prompts us to consider the likelihood that "there is something more important" (2005: 994) going on in the event which is yet to be understood. That is to say, we become open to the possibility of a dramatic redefinition of the parameters of the event.

The second analytic figure is that of the diplomat (Stengers, (2005, 2011). For Stengers, the diplomat is a character that in the cosmopolitical event presents the "voice of whose practice, whose mode of existence and whose identity are threatened by decision" (2005: 1002). In other words, diplomats serve to trouble the usual course of action (whether that be structured by necessity or 'progress', or by 'general interest', or by translation into monetary terms) by evoking how such activities might culminate in an "act of war" (2005: 1003), and thus a calamitous re-ordering of relations. Those who are represented by the diplomat must in principle be committed to the possibility that peace and order will emerge through cosmopolitics: a process that assumes the existence of disparate interests and realities that cannot resolve into a unified state but allowed to co-exist (2011: 375). Key here is that cosmopolitics allows for a suspension of habits that "make us believe that we know what we know and who we are, that we hold the meaning of what makes us exist" (2005: 1003). In other words, diplomats operate by simultaneously invoking the potential of becoming in others involved in an event - such as experts who have second thoughts - but also by advancing the interests or by betraying those who they represent. Additionally, the Diplomat is a figure that seeks to connect different worlds and multiple 
interests, or realities. As such, the diplomat brings about encounters or exchanges between concerned and affected actors and groups in order to engender a peaceful co-existence of multiple modes of being. In sum, the figure of the diplomat allows us to engage with the Bots' complex role in the exchanges that mark the becoming of internally variegated energy-related communities on Twitter.

Lastly, we come to Serres' parasite. This has several connotations though here we focus on its guise as the disruption of a signal relayed between communicator and receiver. In 'The Parasite', Serres (1982a) elaborates on the role of the parasite as an uninvited, and initially disruptive, guest at the dinner table, who exchanges stories for food. On the one hand we can note how the hermeneutic and semiotic are transformed into material (food, shelter) and vice versa; on the other we see how interventions open up the space for new meanings, and how disruption has the potential for generating more complex orders. In parallel to the unruly parasite, is the figure of Hermes (Serres, 1982b) who operates to connect across disparate domains, such as science and myth. However, Hermes is untrustworthy, and the connections and communications he mediates might entail mistranslations and mischief - that is, inject disorder into apparent order.

In summary, these figures facilitate our heuristic efforts at tracing the Bots' impact as they intervene in Twitter communications about energy-demand reduction. The idiot 'urges' a community to redefine itself by attending to nonsensical otherness; the parasite/Hermes draws disparate entities together as a community through a process of exchange, but in the process, introduces noise which makes that community more complex as it re-orders itself; diplomats bring together disparate communities with diverse interests whilst 'respecting their difference'. As such, the figures contribute to a speculative approach to method that builds on the performative capacity of method to shape its object of study. Additionally, these figures serve in the specification of that shaping - a shaping that entails the particular becoming-with of the various elements involved (in the present case, with Twitter) - elements that straddle energy-demand reduction practices, actors and communities.

\section{Exchanges}

With these analytic figures we can begin to discern the various ways in which the Bots insinuate themselves into Twitter-based matters of climate change as well as into the mechanics of Twitter more broadly. In so far as we have adopted a speculative approach, our analysis seeks to access the kinds of becomings that the Bots elicit, something that big data approaches generally fail to address. Each Bot is discussed in sequence to allow our analysis to highlight the ways in which the Bots exhibit different aspects of the analytic constructs discussed above, without being reduced to one figure in particular. This is an important point since through our analysis it becomes apparent that at times the figures (and the Bots) collapse into one another.

\section{@.fimmy_gibbon}

Ostensibly, @Jimmy_gibbon acts like an idiot, translating common sense status updates into short nonsensical texts or murmurings. For example, tweets such as "The traditional means have forgotten the way about the climate change..." or "The scrutiny of confidence of \#wind of the open sea announces the program in order to make the factory of the tu..." evade clear comprehension. Such 
posts also displayed ambivalence and a lack of partisanship for both advocates and sceptics of climate change. That is to say, the posts do not discriminate between the convictions expressed in the messages it posts; @Jimmy_gibbon blindly tweets and retweets environmentally-related posts regardless of political orientation toward energy-demand reduction.

@Jimmy_gibbon thus served to explore Twitter as a medium of entanglement, interference as well as indifference. Here, a variety of users came to the fore including stabilized actors implicated and interested in carbon reduction. The actors could be expert or lay, government or non-government, organizational or individual. Crucially, however, @Jimmy_gibbon also made known non-stabilized carbon reduction actors - that is to say, Twitter users that are: 1) uninterested or ambivalent toward the issue and yet harness the issue as one 'activity' amongst others; 2) actors that in their make-up and capacities are emergent and difficult to pin to fixed ontological categories such as 'human' user or 'organisation'.@Nea1968 and @ClimateFriendly are cases in point. They seem to be indicative of celebrity Bots, organizational Bots or spam related hijacking. In other words,@Jimmy_gibbon began sampling and discerning actors and collectives involved, implicated, or capable of entanglement, in energy-demand reduction or environmental issues and practices on Twitter by way vague and ambivalent posts. In so doing, @Jimmy_gibbon attracted followers and started to receive weekly updates from Twitter containing stories related to keywords contained in @Jjimmy_gibbon's tweets as well the content of its followers. In this respect, @Jimmy_gibbon brings to light Twitter's inbuilt technologies for associating users with trends. On the one hand, those who started to follow @Jimmy_gibbon included users with apparent interest in environmental and climate change issues, such as a U.S. based website ThinkGreenDegrees.com which includes vaguely related information about the sustainability industry but has no discernable focus, The Community Power Report (http://www.communitypowerreport.com) news hub for "project and policy updates", an LED lighting distributor based in Florida, a blogger (http://www.freeenergyplanet.info) posting about technical processes as well as King Tut and Climate Friendly (http://www.climatefriendly.com) an Australian carbon management consultancy, and a Manchester (UK) based plumber interested in renewable energy and sustainable technologies. On the other hand, @Jimmy_gibbon also attracted followers and exchanges with users indifferent to environmental concerns, such as a jobbing U.S. actor and a U.S. army veteran interested in gardening. In one such example, the aforementioned U.S. actor retweeted the post "Australia in talks to link carbon market with..." to seventy-seven followers of his account without qualifying or commenting on the post.

@.Jimmy_gibbon thus served to make visible the emergent actors, collectives and communities, in one way or another implicated in energy-demand reduction by way of exchanges, following and trending, thus sensitising us to the novelty of such entities on Twitter. This complex process of emergence entailed both experts and laypersons as well as organisations, all implicated, by virtue of their communication exchanges, in energy-demand reduction - a process which showed how users can modify and be modified by the collective. So, although@Jimmy_gibbon posts nonsensical messages, such as "Junction Distance - @ homemadelondon where there is to highest soaraway Sunday the sun.co.uk/homepage/t.. bit/ly/xjVnNV", a message such as this gets retweeted by an account ostensibly associated with an Australian model (@Nea1968). Three things seem to be happening here. First, users circulate messages simply to be seen to be active, irrespective of the meaning or relevance of 
the message. Second, as mentioned, users such as @Nea1968 exhibit features of software automation, suggesting that they operate wholly, or in part, through algorithmic processes. Third, the identity and status of users on Twitter are somewhat indeterminate. Another example of software automation came in the form of a direct message from the account @ClimateFriendly, an Anglo-Australian carbon management consultancy: "hey this person is making dreadful posts that are about you..." followed by a link to online malware (malicious software such as viruses). @Jimmy_gibbon attracted many such messages, with the exact same sentence and link, suggesting@ClimateFriendly included automated activity or that the account had been hacked.

Now, despite@Jimmy_gibbon's somewhat senseless murmurings, the Bot attracted followers variously related to environmental issues, as well as followers who display little or no such involvement. Here, followers of @Jimmy_gibbon included: UK based research students studying environmental change and heating installations; renewable heating engineers; energy analysts; two Russian Global energy prize winners in search of renewable energy stories; individual energy and sustainability activists and consultants; community energy-demand reduction initiatives and not-for-profit partnerships; a UK based centre-right think tank; a Florida-based reseller of LED lighting; and many others.

As well as expressing aspects of idiocy, @Jjimmy_gibbon's actions can also be illuminated by way of the parasite where the persistent posting of nonsensical text constitutes a form of 'narrative noise'. @ Jimmy_gibbon produces 'quasi' stories or accounts that add little to the 'substantive' story-telling present in Twitter's energy-related discussions. Despite this, the various modes of exchange occasioned by the Bot (posting, retweeting, appreciations and following) suggest a sort of recruitment metanarrative whereby the 'story' being 'told' or enacted is one of 'numerical force' irrespective of topic or content. In other words, there are simply more users present which adds weight to a conversation or hashtag evoking popularity and significance and thus contributing to the trending of a topic. @Jimmy_gibbon exemplifies how numerical force can include an ambivalence, or indiscrimination, of the relevance of particular users to an issue. Disruptions can thus be subordinated to the numerical magnitude of contributors as an indicator of importance and urgency. Or rather: disorder at one level/scale is subordinated to order at another where the indiscriminate accumulation of followers subsumes the substantive concern of the exchange.

Invariably, such disruptions are neither isolated nor exceptional but are part of the multitude of exchanges mediated by Twitter, not least those in which@Jimmy_gibbon and the other Bots are involved. Thus, the Bots are simultaneously involved in various kinds of exchanges and - by virtue of the timeline of tweets, retweets and conversations - can be seen to emerge out of a chronology of exchanges available in the present. Recalling the observation (Boyd et al., 2010) that Twitter users are simultaneously engaged in multiple conversations, we can suggest that they are also implicated in concatenations, or 'chains' (1982a: 13) of parasitic-host relations (as parasite and/or as host). @Jimmy_gibbon can thus be viewed as both parasite and host - harvesting followers and being followed by others, also acting as parasite/host, thriving on the posts and following of others. What is at play here is the becoming of differing logics and differing interests underpinned by numerical force.

In sum,@Jimmy_gibbon seems to act as a sort of hybrid idiot-parasite that through the nonsensical retweeting of others' posts insinuates itself in the parasitic activities of others. @Jjimmy_gibbon also exemplifies a means for drawing out entities (such as spam Bots and marketing devices) normally 
screened out of other scholars' accounts of microblogging. Finally, @Jimmy_gibbon shows how energydemand reduction becomes an issue that can be subsumed under the interests of other online actors, by way of algorithmic and non-human Twitter Bots.

$$
\text { (a)ecojo1 }
$$

(a)ecojol, by comparison appears to act as a kind of Diplomat/Hermes who tweets and retweets and in doing so occasions links or encounters across disparate users that form 'communities' that are opportunistic, vague and transient. As outlined above, the diplomat involves the processes of discerning the interests, identity and capabilities of actors and translating these into the terms through which exchanges can take place and through which co-existence might be reached within a redefined set of relations. With this in mind, the figure of the diplomat sensitizes us to two key ways in which@ecojol engenders encounters on Twitter. First, @ecojol's exchanges serve to establish the scope of energydemand reduction i.e. the nature and capacity of those involved and their evident commitments and practices. As an upshot of this, @ecojol produces translations of other Twitter users' interests. Second, and like the other bots, @ecojol attempts to produce new ways of thinking about and understanding energy-demand reduction.

To begin to establish the extent of energy-demand reduction on Twitter, @ecojol regularly produced platitudes and truisms as a way of exploring who or what might respond, the types of exchanges that might ensue, as well as what the responses might tell us about others' orientation to the issue. Such expressions included: "So how do we go green?" and "Every day is earth day". On September 30th 2012 @ecojol tweeted “Reduce, Reuse, Recycle.” to which the user@CoreGasLess, a U.S. based manufacturer of an efficient high torque rotary motor, responded “@ecojol So easy and so useful, well said!”. On the $9^{\text {th }}$ of July 2012 @ecojo1 posted "Slowly I will make my money back in energy savings. \#Green Living” to which @ coppertalk, a U.S. based online resource for information about the copper industry, responded "@ecojol Renewable Energy: The Future of America and the Copper Industry." These two examples illustrate how Twitter can serve as a mechanism for commercial organisations engaged in energy markets to promote their interests. Another exchange, however, reveals that energy-demand reduction also involves other kinds of actors. On June $12^{\text {th }} 2012$, (a)ecojol posted the message "Another huge thing a parent can do to live green is to use cloth diapers as opposed to people halfway around the world. Think about it." Which elicited the following response from@indydadblogger: “@ecojo1 True. It's what we do.” Followed by a link to blog post where a husband and father explains the environmental, economic and aesthetic advantages of using cloth nappies. Here, it is not so easy to reduce@indydadblogger's parenting practices to a single broad register, such as environmental action, but rather an engagement with multiple registers simultaneously (cf. Marres and Lezaun, 2011: 496), not least the practices of microblogging as a means to make public and enter into conversation with others.

These platitudes, which frame energy-demand reduction in terms of consumption, can also be understood as invitations to other users to reframe the issues of environmental sustainability and energy-demand reduction in their own terms. This may involve a simple retweet whereby a message is represented as an affirmation of another's activities. Here, @ecojol is retweeted by @elitenetwork_, a 
collective of advisors and installers involved in implementing the UK Government's Green Deal policy to improve the energy-saving capacity of building stock.

七7 Elite Network retweeted you
29 Apr 12: I drive the hybrid, have the solar panels, and live the green life.
Think about it.
29 Apr 12: By living green you could always install solar panels on your
home, invest in wind energy, or purchase a hybrid car.\#eco
Hide other retweets

Figure 1:@ecojol retweeted by Elite Network.

The elicitation of interests also comes by way of Twitter conversations, where a user responds to @ecojo1. Figure 2, for example, shows how the user@GardnFish, a Glasgow-based technology teacher advocating vegetarianism, travel and sustainable living, is seen to promote the practice of home-made cleaning products.

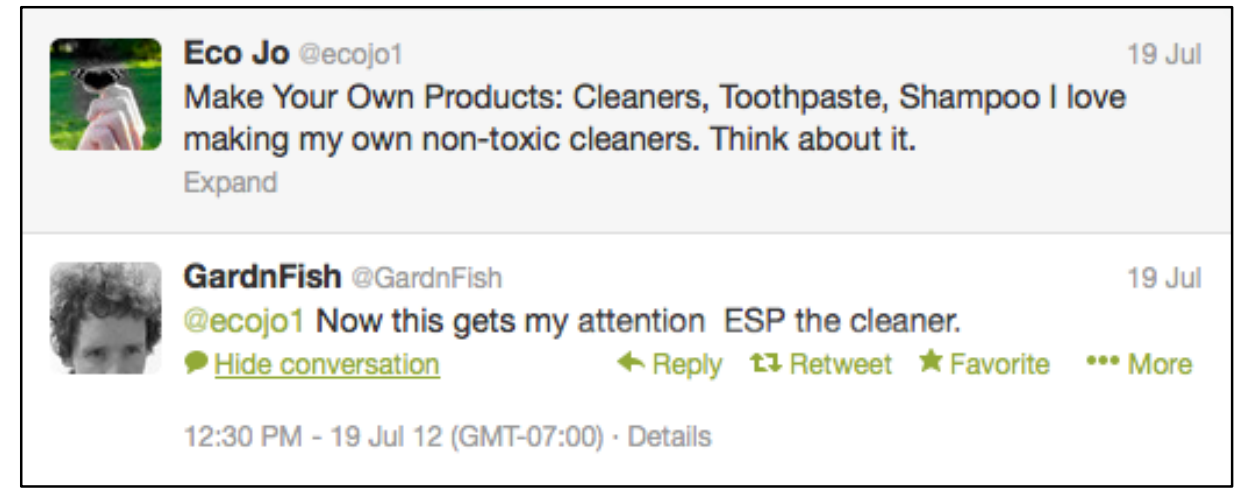

Figure 2: @GardnFish responds to @ecojo1.

(a)ecojol's exchanges with other Twitter users also came in the form of retweets from a variety of different actors. For example, @Health_Plant a UK registered charity and grassroots organisation that promotes green living retweeted "If your workplace doesn’t recycle, suggest they do. Jolly Good." This in turn (see Figure 3) lead to Healthy Planet inviting @ecojol to a community reuse event 'Stuff for Free'. This tweet also included an enquiry as to whether@ecojol had met other green activists and information sources with Twitter presence including Love Food Hate Waste (www.lovefoodhatewaste.com), the Guardian Environment, inhabit (www.inhabit.com) a weblog covering emerging trends in sustainable design and Project Dirt, a London based community that "provides individuals, community groups, companies, organisations and the government (mainly local authorities) a platform to come together and work on tangible green projects."7 


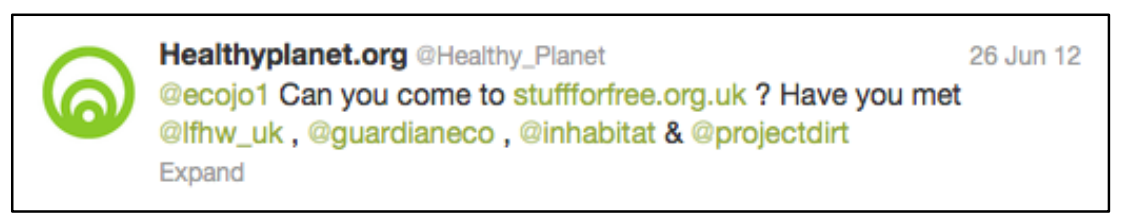

Figure 3: @Healthy_Planet inviting @ecojol to a community reuse event making associations with other users.

Further retweets were circulated by IPAG-CO2; a Canadian NGO that consults on risk assessment associated with carbon storage; an account devoted to marketing the energyEgg® device that automatically turns other electrical devices off, and a London based environmental activist and avowed Christian. Likewise, @ecojol also retweets others' messages, such as those posted by the Welsh office of the World Wildlife Fund (WWF Cymru) or Nigel Pugh of the Canton Green Party, Cardiff.

@ecojol thus serves as the messenger for others in a virtual world where everyone is also messenger. @ecojo1, though, is a relatively undirected messenger whose retweets happen to be visible to the users' followers. As such, @ecojol participates in the making of both online and offline communities (often a mixture of both) where the issue of energy-demand reduction and environmental action is being engaged through all manner of practices and initiatives including product development and marketing, local community efforts or events, and commentary in news media. However, by 'producing' these vague communities, @ecojol as diplomat/Hermes also shows how such collectives are 'made'. This suggests that the invocation of a 'those who are threatened' (Stengers, 2005) is itself highly problematic since 'those' are not necessarily coherent or stable. They may momentarily be pulled into the issue of energy-demand reduction (by way of the translation of reported practices) and may either consent to this translation, or contradict it. The diplomatic role of @ecojol is thus ontologically ironic - it at once represents those who are threatened, makes 'those', and betrays them by revealing their private concerns and/or disinterestedness. In this sense, betrayal is a trait of all three bots in that they elicit responses that serve to unmask the interests, agendas and mechanics of other users, most notably those evidencing algorithmic aptitudes, clandestine or tangential interests or those that are simply duplicitous. On this score, @ecojol can also point to a re-thinking of, or expansion of, , the figure of the diplomat.

Arguably, then, and viewed through the figure of the diplomat, @ecojol is rendering the complexity of energy-demand reduction and how it is variously practiced, not least by way of Twitter. Here, UK policy instruments for addressing the environmental practices of UK citizens are set alongside a much wider variety of practices where energy-demand reduction is but one of a number of means for addressing sustainability and environmental action. Another way of putting this, drawing on Latour's (2007) typology of the political, is that @ecojol illuminates Twitter as a medium in which various kinds of climate change politics are being enacted simultaneously. These range from political-1, where new associations are being forged between emerging and existing actors, through to political-5, where the environment is an object of state intervention and energy-demand is represented as an established political-economic issue (not least via advisors and installers such as the Elite Network). Thus, @ecojol becomes a kind of envoy that passes through different enactments of carbon-reduction as a modality of sustainable living. This passage involves encounters and exchanges with actors who are themselves orienting to stabilized and/or emerging relations of climate change. As Stengers notes 
(2011: 376), employing terms drawn from Deleuze and Guattari, the diplomat passes between - indeed, exposes the co-existence of - "territorialization" and "deterritorialization". Here, we see@ecojol tentatively tracing a variety of collectives, not least those involved in: market driven efforts to capitalize on sustainability; the mediation of government efforts to implement concrete energy-saving fixes; emerging grassroots and local communities instructing people on material practices for engaging with sustainability; and, in the case of @GardnFish, individuals who are seemingly open to changing their practices. In all this, @ecojol usefully reveals how emergent communities can also be 'usurped' by other users who use them direct attention towards their own non-environmental ends.

Insofar as@ecojol has played the role of diplomat, it has explored how those implicated in energydemand reduction have concerns that can differ and resonate, and engage in practices that diverge yet overlap: and all this does not necessarily result in debilitating contradiction and conflict. Or, in other words, @ecojol intervenes to show how Twitter entails multiple interacting norms, a multitude of crosscutting practices, and a plethora of partially connected collectivities, more or less associated with the environment.

\section{a)ErtBot}

In contrast to the other Bots, @ErtBot is programmed to elicit different modes of exchange (see Figure 4). @ErtBot explicitly and mechanically detects and construes accounts of technological practices as instances of energy consumption and therefore environmental action. As such, Twitter users are encouraged to reflect on their engagement with climate change and post their response.

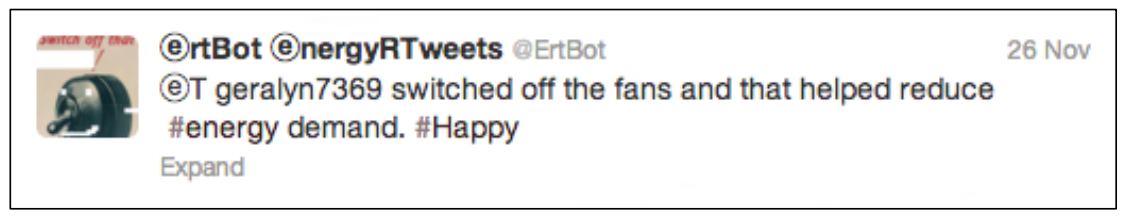

Figure 4: A typical @ErtBot translation of technological practices as implicated in energy consumption.

@ErtBot thus translates everything into the vocabulary of energy-demand reduction. However, the constancy of this translation means that it not really attuned to other typical meanings of the events it is responding to. An exchange with the user@@_badbadmood is illustrative. @ErtBot's tweet “@T millaayrevels switched off the computer and that helped reduce our collective \#energyDemand" is followed by the users' response “@ErtBot I feel so bad omg I put it on standby IM SO SORRY SWITCHING OFF PROPERLY NOW”.@_badbadmood banters with@ErtBot and emphasizes, with caps lock, genial compliance with the suggestion that doing so is an environmental act. 


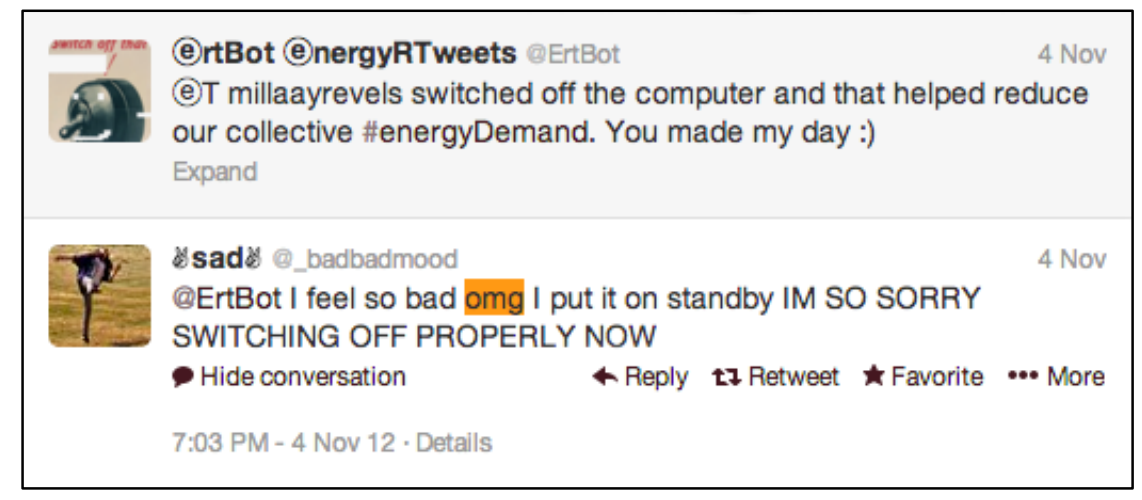

Figure 5: @_badbadmood promising to switch off properly. ${ }^{8}$

Other typical exchanges include expressions of indignation with the assertion that their practices have environmental repercussions. For instance, @olugly responds to @ErtBot’s suggestion that non-access to the Internet is beneficial for the environment by stating "shut up its not good news for me" (reflecting the reported fact her mobile phone has been confiscated). In resisting @ErtBot's energy-related reframing@oiugly refuses to be mobilized.

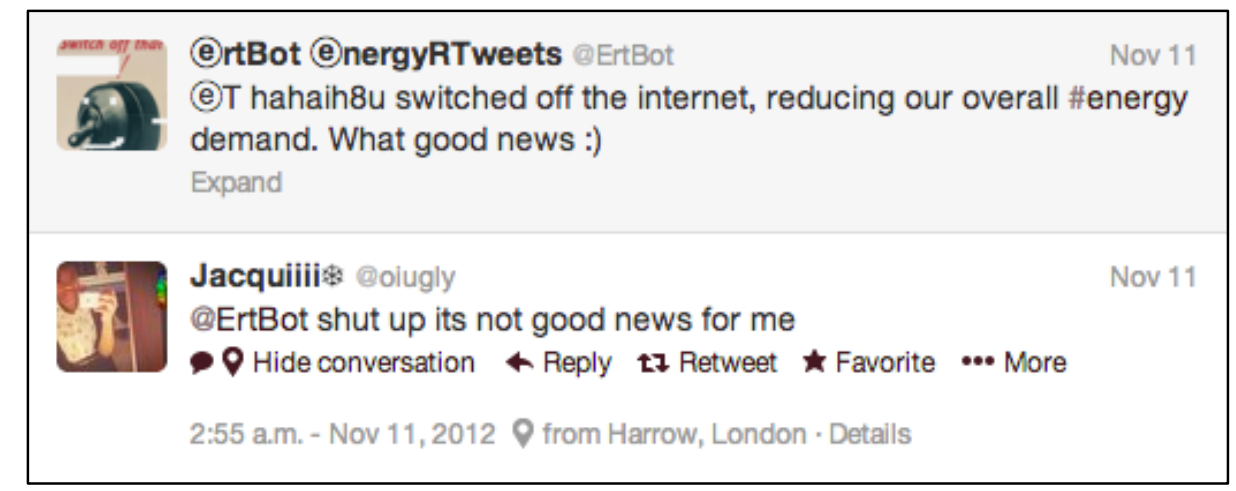

Figure 6: @olugly refusing @ErtBot's translation.

In contrast to exchanges that address environmental implications, other exchanges elicit a different order of response. For example, @FrodoAllardice, responding to @ErtBot's assertion that switching off Christmas might help to reduce energy demand simply rebuffs @ErtBot, stating that it is "nonsense". Like@ecojo1,@ErtBot has diplomatic traits insofar as it invites other users, whose reports feature device-related actions, to reflect on their practices through the lens of energy-demand reduction. @ErtBot thus invites users to consider the public risks and obligations of their actions (even when these are accidental) while problematizing their private concerns or interests. Arguably, then, @ErtBot provokes a certain mode of becoming-with, where those users not configured as participants in energydemand reduction are offered the opportunity to be refigured as interested actors. Thus, @ErtBot enacts the potentiality that the question of energy-demand reduction applies to the ostensibly disinterested or disengaged users.

Akin to@Jimmy_gibbon, @ErtBot also evinces idiocy in that its automated utterances often simply do not make sense. This can be illustrated by thus. "AvSats switched off the tv and that helped reduce 
\#energy demand. Lovely." [sic] typifies an intelligible post, whereas "switched off the internet", "switched off the brain", and "switched off the Christmas" are examples of idiotic utterances. This, however, demonstrates that any incident can be translated into energy-demand reduction and thus, it begins to crystallize the idiocy of such a 'generic' translation. That is to say, it makes us wonder why it is that any event can be so readily translated into the discourse and practices of energy-demand reduction, and whether those events that are routinely articulated in terms of energy-demand reduction might be fruitfully and inventively enacted in other ways. Twitter users' responses to the idiocy of @ErtBot's interventions can be seen to broadly fall within two modes of exchange. First, there are those users who simply deny @ErtBot's translation of their practices thus re-drawing the parameters of the issue. Second, there are those users who insist that there are more important things going on of which @ErtBot is unaware. In both cases, these responses orient us as analysts, and potentially the respondents themselves, toward a slowing down and re-consideration that there may be other things going on, other parameters in play, and other issues at stake that cannot be assimilated to energydemand reduction.

\section{Concluding remarks}

In this paper, we have drawn on performative approaches to methodology to develop a speculative method. This has been derived from speculative design in general, and the ECDC speculative design (and ethnographic) project in particular. In the process, we have designed and deployed preprogrammed Bots playfully to intervene in and provoke the Twitter-sphere, a key site where energy politics are played out. Our aim has been to use the Bots to trace how, within the dynamics of Twitter, communications circulate, communities are enacted, and identities are rendered in relation to energydemand reduction. More importantly, in their oblique, ludic and nonsensical aspect, the Bots were meant to explore the emergent and prospective elements of this domain of Twitter - to engage with the 'virtual of the virtual', so to speak. The Bots' actions on Twitter were analysed with the aid of three heuristic conceptual characters. Provisionally, we can say that this exercise showed how, for instance: (energy-demand reduction) 'community' can emerge as much a result of the indiscriminate accumulation of communications and followers, as of explicit, shared content; at the same time, users (and the Bots) are transient and unreliable conduits of communication that simultaneously tie together and undo 'communities', that enact moments of similarity and difference amongst themselves and other users; the excessive translation of everyday activities and events into the terms of energy-demand reduction opens a prospective space for querying those very terms, the parameters of their application, and alternatives to them.

Taken together the Bots probe the proliferation and propagation of new political configurations mediated by Twitter where environmental concerns, practices, technologies and actors are in constant flux - tentative, emerging, and (dis)organising. Thus, the Bots engage Twitter as a mode of what Latour (2007: 816) calls 'Political-1' in which each new association changes the collective and the community. Moreover, the sheer openness and complexity in the way that energy-demand reduction is enacted on Twitter suggests that there are other ways of doing politics. Thus, the evident ambivalence and disinterestedness of actors on Twitter does not necessarily equate with total disengagement. That 
environmental collectives are eventuated through numerical magnitude, can be made and remade, can be simultaneously singular and multiple, and can potentially have a 'right' or 'apt' temporal moment (or kairos), in which collective engagement can be cursory or scant, implies new ways of thinking about environmental engagement and its prospects.

Finally, we return to the issue of speculative methodology. In this paper we have developed and deployed a very particular version of this to explore the possibilities latent in the Twitter-sphere's enactments of energy-demand reduction. Needless to say, there are many other techniques - not least derived from design and the arts - available that can do similar things. Speculative methodology, of course, is not descriptive: it is performative. Its interventions are designed to 'prompt' (as much as probe) emergent enactments that can problematize existing practices (in our case, the neat conceptualization of community and the practices of energy-demand reduction) and open up the prospective. As such, this is a form of methodology that, as Stengers (2010: 57) puts it, "affirms the possible, that actively resists the plausible and the probable targeted by approaches that claim to be neutral". However, there is no guarantee that speculative interventions and devices will 'affirm the possible'. They can be grossly alienating as well as playfully confusing, or obliquely inviting: they can, in other words, just as easily precipitate a flight into 'the plausible and the probable' by the actors who are being speculatively engaged. Put another way, the speculative attaches no less to the viability of speculative devices and interventions themselves. There is, therefore, no guarantee that speculative devices and their provocations will work - experiments can and do fail.

${ }^{1}$ For Ruppert et al. (2013: 15) the term 'big data' points to the routine and increasingly intensive production, storage and analysis of commercial, scientific and governmental data sets, the proliferation of data sources and resources required to support big data practices, such as computational and processing technologies and analytic tools.

2 See http://www.esrc.ac.uk/news-and-events/press-

releases/3400/Using_communities_to_find_the_answers_to_energy_demand_problems_.aspx, Date accessed 29th March, 2013. Project code: ES/1007318/1.

${ }^{3}$ For UK policy efforts to address climate change through carbon reduction see (Dept. of Energy and Climate Change, 2009a, 2009b).

${ }^{4}$ Examples of controversies triggered on Twitter include: the trial of Paul Chambers in the UK for tweeting purportedly threatening intentions to blow up Robin Hood airport, closed due to snow, "sky high!!"; a defamatory message posted by Sally Bercow, wife of the Speaker of the House of Commons, intimating Lord McAlpine's involvement in a child abuse scandal; anonymous tweets implicating a UK footballer in an extra-marital affair despite the existence of a 'super-injunction'.

5 Together with regulatory and economic measures, 'behavior change' (e.g. Dietz et al., 2009) is currently a key UK environmental policy objective, where a benign informational 'nudge', such as the introduction of smart monitors in the home, is considered an effective intervention into citizens' and populations' routine energy demands (Darby, 2006). Recent scholarship, however, has begun to refute the efficacy of behavioral intervention as well its very premise. On the one hand, behavioral intervention can result in a 'boomerang effect' where energy saving technologies actually lead to an increase in consumption; on the other, the very premise of behavior change is flawed give the very considerable complexity of energy consumers (Strengers, in press) and their sociocultural settings and practices (Shove, 2003).

${ }^{6}$ For an in-depth description of the Bots see: http://www.ecdc.ac.uk/2013/08/07/our-bots/

7 http://www.projectdirt.com/pages/about-us/ Date accessed Wednesday, July 10, 2013. 
${ }^{8}$ Curiously, as Figure 5 shows,@_badbadmood seems to have changed username from millaayrevels since@ErtBot first posted illustrating the indeterminacy and mutability of users' identity on Twitter.

\section{Acknowledgements}

This paper draws on research conducted under the project grant 'Sustainability Invention and Energydemand reduction: Co-designing communities and practice' funded by RGUK and led by the EPSRG (project code ES/1007318/1). The authors would like to thank the members of the project team including Bill Gaver, Tobie Kerridge and Liliana Ovalle.

\section{$\underline{\text { References }}$}

Adkins, L. and C. Lury (eds) (2012) Measure and Value. Malden, MA; Oxford; Carlton, Victoria: WileyBlackwell.

Back, L. and N. Puwar (eds) (2012) Live Methods. Malden, MA; Oxford; Carlton, Victoria: WileyBlackwell.

Boyd, D. et al. (2010) 'Tweet, Tweet, Retweet: Conversational Aspects of Retweeting on Twitter', pp. 1-10 in System Sciences (HICSS), 2010 43rd Hawaii International Conference on.

Boyd, D. and K. Crawford (2012) 'Critical Questions for Big Data', Information, Communication Ë Society 15(5): 662-79.

Burns, A. and B. Eltham (2009) 'Twitter Free Iran: An Evaluation of Twitter's Role in Public Diplomacy and Information Operations in Iran's 2009 Election Crisis'.

Crompton, R. (2008) 'Forty Years of Sociology: Some Comments', Sociology 42(6): 1218-27.

Darby, S. (2006) 'The Effectiveness of Feedback on Energy Consumption', A Review for DEFRA of the Literature on Metering, Billing and Direct Displays 486.

Dept. of Energy and Climate Change (2009a) The UK low carbon transition plan: national strategy for climate and energy.

Dept. of Energy and Climate Change (2009b) The UK renewable energy strategy.

Dietz, T. et al. (2009) 'Household Actions Can Provide a Behavioral Wedge to Rapidly Reduce US Carbon Emissions', Proceedings of the National Academy of Sciences 106(44): 18452.

Fraser, M. (2010) 'Facts, Ethics and Event', pp. 57-82 in Jensen, C.B. and Rödje, K. (eds), Deleuzian Intersections: Science, Technology and Anthropology. New York; Oxford: Berghahn Books.

Gaver, W. (2002) 'Designing for Homo Ludens', I3 Magazine 12: 2-6.

Gerlitz, C. and B. Rieder (2013) 'Mining One Percent of Twitter: Collections, Baselines, Sampling', $M / C$ Journal 16(2).

Goolsby, R. (2009) 'Lifting Elephants: Twitter and Blogging in Global Perspective', pp. 1-6 in Young, M.J. et al. (eds), Social Computing and Behavioral Modeling. URL (consulted April 2013): http://link.springer.com/chapter/10.1007/978-1-4419-0056-2_2

Honeycutt, C. and S.C. Herring (2009) 'Beyond Microblogging: Conversation and Collaboration via Twitter', pp. 1-10 in System Sciences, 2009. HICSS'09. 42nd Hawaï International Conference on.

Hughes, A.L. and L. Palen (2009) 'Twitter Adoption and Use in Mass Convergence and Emergency Events', International Fournal of Emergency Management 6(3): 248-60.

Java, A. et al. (2007) 'Why We Twitter: Understanding Microblogging Usage and Communities', pp. 56-65 in Proceedings of the 9th WebKDD and 1st SNA-KDD 2007 Workshop on Web Mining and Social Network Analysis, WebKDD/SNA-KDD '07.

Latour, B. (2007) 'Turning Around Politics: A Note on Gerard de Vries' Paper', Social Studies of Science 37(5): $811-20$.

Law, J. (2004) After Method: Mess in Social Science Research, International Library of Sociology. London: Routledge.

Lezaun, J. et al. (2013) 'Provocative Containment and the Drift of Social-Scientific Realism', Fournal of Cultural Economy 6(3): 278-93.

Lezaun, J. and L. Soneryd (2007) 'Consulting Citizens: Technologies of Elicitation and the Mobility of Publics', Public Understanding of Science 16(3): 279-97.

Lury, C. and N. Wakeford (2012) Inventive Methods: The Happening of the Social. London; New York, NY: Routledge.

Marres, N. and J. Lezaun (2011) 'Materials and Devices of the Public: An Introduction', Economy and Society 40(4): 489-509. 
Marres, N. and R. Rogers (2005) 'Recipe for Tracing the Fate of Issues and Their Publics on the Web', pp. 922-35 in Latour, B. and Weibel, P. (eds), Making Things Public: Atmospheres of Democracy. Karlsruhe; Cambridge, Massachusetts; London, England: ZKM; The MIT Press.

Marres, N. and E. Weltevrede (2012) 'Scraping the Social? Issues in Real-Time Social Research', Fournal of Cultural Economy: 1-52.

Michael, M. (2004) 'On Making Data Social: Heterogeneity in Sociological Practice', Qualitative Research $4(1): 5-23$.

Michael, M. (2012a) 'Toward an Idiotic Methodology: De-Signing the Object of Sociology', Sociological Review 60(s1): 166-83.

Michael, M. (2012b) "WWhat Are We Busy Doing?" Engaging the Idiot', Science, Technology Ë Human Values 37(5): 528-54.

Michael, M. and W. Gaver (2009) 'Home Beyond Home Dwelling With Threshold Devices', Space and Culture 12(3): 359-70.

Murthy, D. and S.A. Longwell (2012) 'Twitter and Disasters', Information, Communication \& Society: 1-19.

Rogers, R. (2009) The End of the Virtual: Digital Methods. Amsterdam: Vossiupers UvA.

Rogers, R. (2010) 'Internet Research: The Question of Method-A Keynote Address from the YouTube and the 2008 Election Cycle in the United States Conference', Journal of Information Technology \& Politics 7(2-3): 241-60.

Rogers, R. (2013) Digital Methods. MIT Press.

Ruppert, E. (2011) 'Population Objects: Interpassive Subjects', Sociology 45(2): 218-33.

Ruppert, E. et al. (2013) 'Reassembling Social Science Methods: The Challenge of Digital Devices', Theory, Culture \& Society.

Ruppert, E. and M. Savage (2011) 'Transactional Politics', The Sociological Review 59(s2): 73-92.

Savage, M. and R. Burrows (2007) 'The Coming Crisis of Empirical Sociology', Sociology 41(5): 885-99.

Serres, M. (1982a) The Parasite, Posthumanities. Minneapolis, London: The Johns Hopkins University Press.

Serres, M. (1982b) Hermes: Literature, Science, Philosophy. Johns Hopkins University Press.

Shah, C. and C. File (2011) 'InfoExtractor - A Tool for Social Media Data Mining', FITP 2011: The Future of Computational Social Science.

Shove, E. (2003) Comfort, Cleanliness \&̊ Convenience, New Technologies/New Culture Series. Oxford; New York: Berg.

Shove, E. et al. (2012) The Dynamics of Social Practice. London; Thousand Oaks, CA; New Delhi; Singapore: Sage Publications. URL (consulted March 2013): http://www.uk.sagepub.com/books/Book235021?prodId=Book235021

Stengers, I. (2005) 'The Cosmopolitical Proposal', pp. 994-1003 in Latour, B. and Weibel, P. (eds), Making Things Public. Cambridge, MA: MIT Press.

Stengers, I. (2010) Cosmopolitics I. University of Minnesota Press.

Stengers, I. (2011) Cosmopolitics 2, Posthumanities (Vol. 2). Minneapolis; London: University of Minnesota Press.

Strengers, Y. (in press) Smart Technologies in Everyday Life. Basingstoke: Palgrave Macmillan.

Thrift, N. (2005) Knowing Capitalism. London: SAGE Publications.

Tumasjan, A. et al. (2010) 'Predicting Elections with Twitter: What 140 Characters Reveal about Political Sentiment', pp. 178-85 in Proceedings of the Fourth International AAAI Conference on Weblogs and Social Media.

Webber, R. (2009) 'Response to 'The Coming Crisis of Empirical Sociology': An Outline of the Research Potential of Administrative and Transactional Data', Sociology 43(1): 169-78.

Weltevrede, E. and A. Helmond (2012) 'Where Do Bloggers Blog? Platform Transitions within the Historical Dutch Blogosphere', First Monday 17(2).

Wenger, E. (1998) Communities of Practice: Learning, Meaning and Identity. Cambridge: Cambridge University Press.

Whitehead, A.N. (1978) Process and Reality: An Essay in Cosmology. (Gifford Lectures of 1927-8; corrected edition eds. Griffin, D and Sherburne, D.). New York: The Free Press. 